Teasley, S.D., Finholt, T.A., Potter, C.S., Carragher, B., Carroll, L., Conway, C., Grosser, B., Hanlon,

J., Kisseberth, N., Robinson, S., Stone, D., Thakkar, U., Weber, D., Snow, G.R., Claes, D.R.,

\& Myers, J.D. (2000). Participatory Science via the Internet. In B. Fishman \& S. O'Connor-Divelbiss (Eds.),

Fourth International Conference of the Learning Sciences (pp. 376-383). Mahwah, NJ: Erlbaum.

\title{
Participatory Science via the Internet
}

\author{
Stephanie D. Teasley (Chair), Thomas A. Finholt (Presenter) \\ University of Michigan, CREW, 1075 Beal Ave., Ann Arbor, MI 48109-2112 \\ Tel: 734-647-4948, Fax: 734-936-3168 \\ Email: steasley@umich.edu, finholt@umich.edu
}

\begin{abstract}
Clint S. Potter (Presenter), Bridget Carragher, Liana Carroll, Charles Conway*, Benjamin Grosser, Janet Hanlon, Nick Kisseberth, Scott Robinson, David Stone*, Umesh Thakkar** and Daniel Weber Beckman Institute, **National Center for Supercomputing Applications and *University High School, University of Illinois at Urbana-Champaign, Urbana, IL 61801

Tel: (217) 244-1958, Fax: (217) 244-6219

Email: cpotter@uiuc.edu

Gregory R. Snow (Presenter) and Daniel R. Claes

University of Nebraska, Dept. of Physics \& Astronomy, 116 Brace Hall, Lincoln, NE 68588-0111

Tel: 402-472-6279. Fax: 402-472-2879

Email: gsnow@unlhep.unl.edu

James D. Myers (Presenter)

Pacific National Laboratory, Environmental Molecular Sciences Laboratory, 3335 Q St., Richland, WA 99352

Tel: 509-376-9558, Fax: 509-376-0420

Email: jim.myers@pnl.gov
\end{abstract}

\begin{abstract}
This special session features scientists and developers building innovative interactive virtual environments that can be used for science education from kindergarten through college. The four projects presented in this session focus on different areas of science, from space physics to entomology, but they share a common educational orientation towards participatory learning using Internet access to scientific instruments, data, and expertise.
\end{abstract}

Keywords: distributed learning environments, science education

\section{Introduction}

There is an increasing emphasis in science education to provide learners with opportunities to "do science" in the classroom in ways that are representative of how scientists themselves work. Much of the effort at providing such experiences centers around the use of technology-supported learning environments that model or simulate real world scientific practice (e.g., Horwitz et al., 1998; Edelson, 1998). In contrast, this special session features scientists and developers building innovative interactive virtual environments for scientists which are then also used for science education from kindergarten through college. The four projects presented in this session focus on different areas of science, from space physics to entomology, but they share a common educational orientation towards participatory learning using Internet direct access to scientific instruments, data, and expertise. In each case, students are provided with tools for engaging in scientific activity that are intended to be personally meaningful, cognitively complex, and socially situated within authentic scientific practice.

Science takes place traditionally in laboratories where the conduct of the research is centered around the direction of a "chief-scientist-knower" (Mukerji, 1996). In this model, the practice of science depends on residence in a lab for exposure to domain expertise, as well as for access to specific equipment needed for the research. In recent years, collaboratory and other Internet-based projects have demonstrated the feasibility and scientific usefulness of using the Internet to link teams of people, data, tools, and facilities to overcome barriers of time and distance (Finholt \& Olson, 1997). For example, supercomputers at the University of California-San Diego couple a high-voltage electron microscope with a data-intensive computational environment to allow remote access for capturing, analyzing, and creating 3D models of anatomical tissue specimens (Young, Guo You Fan, Hessler, \& Lamont, 1996). As there are very few of these microscopes and access to supercomputers are rare outside of the computer science world, this collaboratory project turns the microscope and associated image processing and 
analysis software into a network resource available to researchers in any laboratory via the Internet. The adoption of such technology has the potential to radically alter how scientists work in the digital age by freeing them of the social and physical constraints of the traditional laboratory.

The new technology supporting distributed work will undoubtedly change the practice of science, and it can also bring related changes to the training of budding scientists at all educational levels. One of the features of many collaboration tools is the increased visibility of communication between users or between users and instruments. For example, some collaboratories employ chat rooms for scientific discussion in the absence of any good vehicle for multi-party voice over Internet. "Observation" of these chat room discussions allow scientists-intraining to turn what might otherwise be considered lurking behavior into the more acceptable opportunity for what Lave calls "legitimate peripheral participation" (Lave \& Wenger, 1991). Specifically, following the ongoing chat discussion between several senior scientists as they discuss incoming data allows more junior colleagues (e.g., undergraduate students) to see how interpretations of the scientific findings get negotiated and agreed upon. This is an experience that they might not otherwise have, or have much later in their training. In addition, tools designed for scientists to do remote data collection also allow new opportunities for learning as students can have access, either observationally or by actual manipulation, to expensive and/or scare instruments that have previously been a resource for only a few select researchers. The exposure to ongoing scientific discourse and access to real scientific tools-in-use afford students with a kind of authentic learning experience that extends classroom science inquiry beyond traditional boundaries (e.g., Barstow, 1998).

\section{The Collaboratory as Classroom: Challenges and Benefits of Online Participatory Science Education (Finholt)}

Global computer networks are the setting for collaboratories, or computer-supported "laboratories without walls," where scientists can work with each other, facilities, and databases independent of geographic location (National Research Council, 1993). This paper explores how collaboratories create new opportunities for participatory science education. Specifically, the Space Physics and Aeronomy Research Collaboratory (SPARC) (1), a prototype electronic environment for conducting observations of the sun and of the earth's upper atmosphere, provides unprecedented access by undergraduate and graduate students to authentic research activity in space physics (Finholt \& Olson, 1997).

\section{The Scope and Design of SPARC}

Traditionally, training in space physics has provided only rare occasions for students to join with experienced scientists in key research tasks, such as data collection. For example, a major impediment to shared activity is the remoteness of instruments and observatories, and the high cost of visiting these sites (e.g., Greenland, Antarctica etc.). With SPARC, this constraint is relaxed, since activity occurs online. Today, SPARC offers real time views of over 150 ground-based instruments (e.g., incoherent scatter radars), space-based instruments (e.g., UV imagers on satellites), and supercomputer models (e.g., the Thermosphere-Ionosphere Nested Grid model).

Since 1992, the SPARC system has progressed through several development stages. The current phase, starting in late 1998, reflects a "thin client" strategy where users require only an Internet connection and a Web browser to view data visualizations. Figure 1 illustrates important features of the SPARC interface. The lower lefthand corner is a persistent chat window, where users can discuss unfolding atmospheric events. The middle panel on the left indicates who else is viewing the current page. The upper right hand corner shows two views of the earth from polar orbiting satellites, and one view of the sun. The display in the middle of the page shows current time series data plotting electron density VS. altitude from an incoherent scatter radar in Massachusetts. The plots at the bottom are measures of interplanetary magnetic fields, force of the solar wind and so forth - as captured by the ACE satellite.

\section{New Opportunities for Participatory Science}

Because SPARC affords interaction independent of time and location, students have had earlier and deeper exposure to space physics research practices and to practitioners. A key finding is that SPARC improves access by students to knowledge within a scientific community. For example, Figure 2 illustrates the pattern of interaction during an intensive data collection effort (called a "campaign" by space scientists). In this campaign, two teams of scientists interleaved their activity via SPARC over several days. Note in particular the communication ties between 


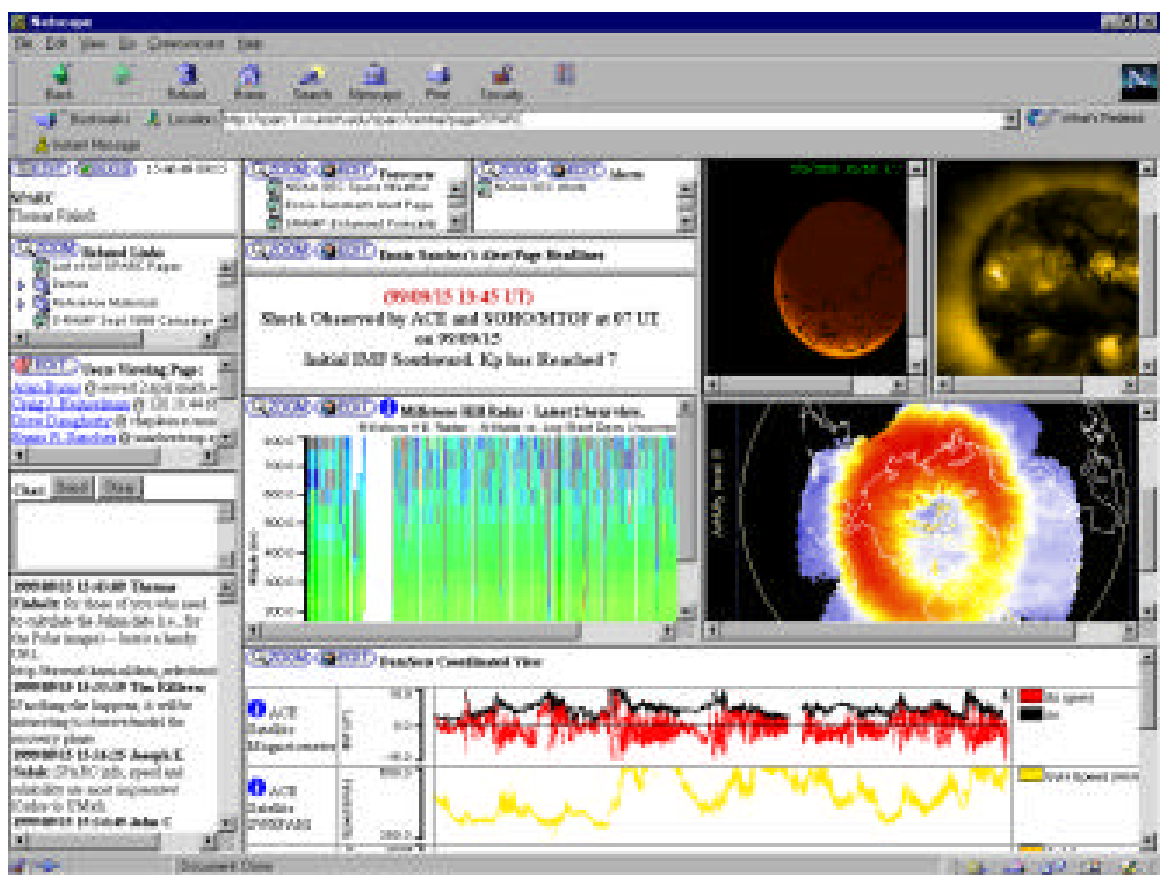

Figure 1. The SPARC interface

the undergraduates at Florida Tech and experienced scientists at Michigan and at SRI International (located in Menlo Park, CA). Analysis of the content of these interactions shows that the Florida Tech students were receiving important direction and mentoring from the remote scientists - beyond what local scientists provided. In addition, each student had a chance to control the ongoing experiment. This authentic exposure led one student to observe (speaking of upper atmospheric phenomena) "In class these were just equations - but now I can actually see the effects."

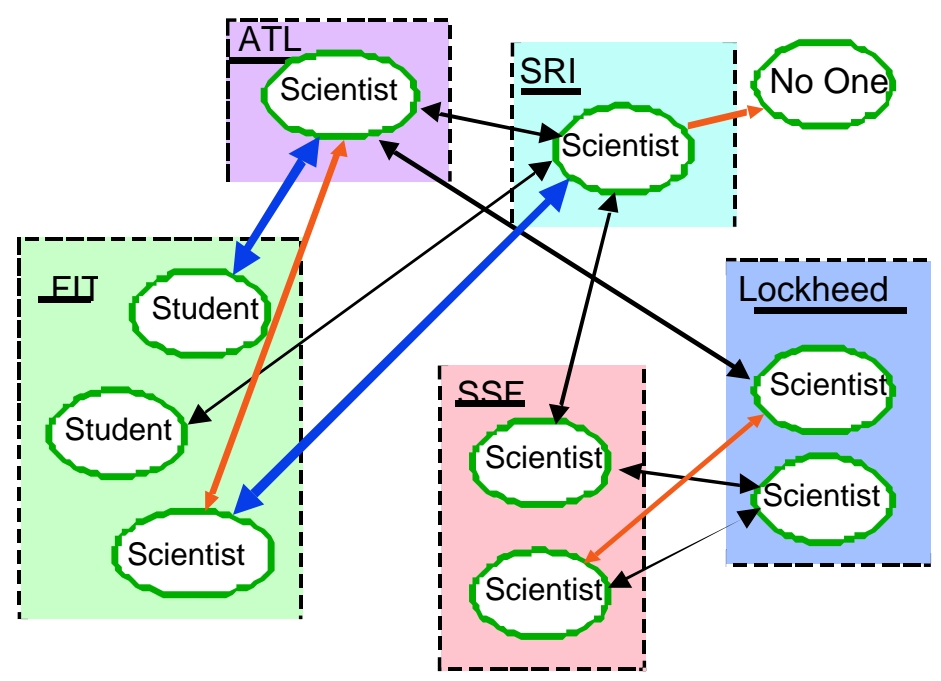

Figure 2. Interaction by students during a SPARC campaign 


\section{SPARC Impact}

SPARC and other collaboratories create new arenas for learning by expanding opportunities, especially for students, to join in with experienced scientists in the conduct of research projects. However, this new style of participatory education may require teaching and mentoring skills beyond the demands of familiar lecture and labstyle learning. In SPARC, senior scientists were particularly motivated to mentor students because the collaboratory was novel. It is unknown how willing busy researchers will be to take time for novices as mechanisms for Internetmediated science become more common. Within SPARC, attempts have already been made to establish a middle ground where very young scientists, such as elementary and middle school classes, can view atmospheric events with coaching from an experienced researcher. This strategy transmits some of the excitement of participating in the unfolding research activity, without compromising attention and communication resources among the principal investigators.

\section{Bugscope: A Sustainable Remote Microscope Project for K-12 (Potter et al.)}

Bugscope (2) is a second-generation educational project in the WorldWide Laboratory (Potter \& Carragher, 1999) that provides web browser based control of scientific imaging instrumentation using the Internet. We had previously demonstrated web based remote access to sophisticated scientific imaging systems several years ago in the Chickscope project (3) (Bruce et al., 1996). The primary goal of the Bugscope project is to demonstrate that relatively low cost, sustainable access to an environmental scanning electron microscope (ESEM) can be made available to K-12 classrooms.

\section{Methods}

To participate in the project, a classroom submits a web-based application that describes how they plan to use the microscope. If the application is accepted, a one hour session on the ESEM is scheduled and the classroom mails in their chosen specimen. During their access time, classrooms use a standard web browser over the Internet to control and acquire images from the ESEM (Philips/FEI XL-30FEG). The interface is shown in Figure 3 and the classroom can control stage movement, magnification (10-10,000x), focus and contrast. Images, including any annotations and comments made by the participants, are automatically stored to a database along with the associated acquisition parameters. The database is immediately accessible from the Bugscope web site to any interested observer. The software allows for several classrooms to simultaneously and independently access and control the microscope thus providing the potential for supporting multiple classrooms during each session. The effectiveness of the Bugscope project is evaluated by requesting background information from the classroom teacher prior to the project, feedback immediately following the session, and a project report a week after the session.

To achieve the goal of low cost, sustainable access to the instrument, it is clear that professional university staff can not be involved in the weekly operational aspects of the project. In the Bugscope project this problem has been addressed by training a team of high school students and undergraduates to prepare the specimens and perform the initial microscope setup. Another key aspect of sustaining the project has been to automate many of the required project administration and data handling tasks. An infrastructure has been developed so that applications submitted by the teachers through the Web are automatically archived into a database. These are then reviewed and scheduled, using a Web based interface, by the project team and the results are automatically collected to provide an overall merit value for the proposed project. This information is then used to set up and schedule a session for the classrooms. During the session the acquired images are also automatically stored and can be later retrieved by the classroom (or any other interested group) though the Web. Without this automation, sustaining this project in the long term would be overwhelming and would require the assistance of a full time project administrator. 

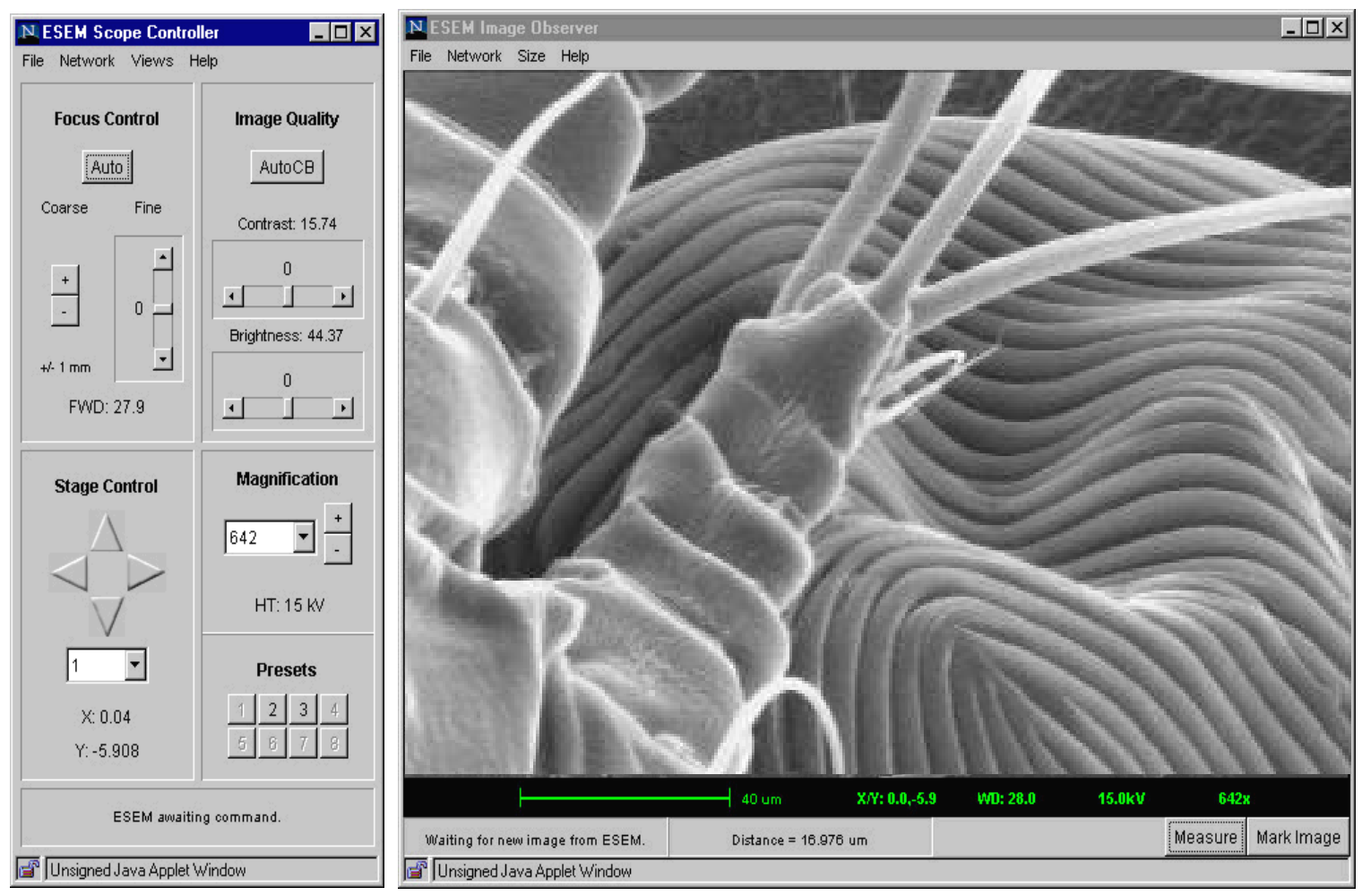

Figure 3. The IRMA Microscope Controller (left) and Image Observer (right).

\section{Results and Conclusions}

As of February 2000, we have supported over 30 classrooms (approximately 1000 students) from all over the country. We have had enthusiastic response from the students and teachers that have participated in the project and over 100 applications have been submitted. We currently routinely support two classrooms a week and we expect to support approximately 100 sessions a year. The project requires minimal staff resources and less than 3 hours of instrument time a week.

The most obvious benefit of the Bugscope project is that is provides students with access to scientific instrumentation and expertise that would not otherwise be available to them. More importantly the project engages students in the scientific process and gives them some experience of the realities of conducting scientific research. In planning the experiment and providing the specimens, students have a sense of ownership of the project that would be very difficult to duplicate using a simulator or a virtual machine. This benefit was pointed out numerous times in the final evaluations submitted by the participating teachers.

\section{The Cosmic Ray Observatory Project (CROP): A Coordinated Network of High School Based Experiments Studying Cosmic-Ray Showers in Nebraska (Snow)}

The Cosmic Ray Observatory Project (CROP) (4) is a statewide education and outreach experiment whose goal is to involve Nebraska high school students, teachers, and college undergraduates in a multi-faceted, hands-on research effort to study extended cosmic-ray air showers. High-energy cosmic rays striking the atmosphere create avalanches of particles which can cover up to 50 square miles at the earth's surface. Particles from extended showers will be detected by arrays of 24" x 24" plastic scintillation counters placed in weather-proof enclosures on high school rooftops, while a PC-based data-acquisition system located inside the school building records events. A GPS receiver will provide a time stamp so that time coincidences with other sites, signaling the presence of extended cosmic-ray showers, can be detected.

\section{Methods}

Student participants will compare data with other sites via the Internet and share experiences through regional workshops and videoconferences organized around the state's 19 Educational Service Units (ESUs). Successful measurements require all existing detector sites to be collecting data simultaneously. A pilot phase will 
outfit selected sites in the nearby Lincoln/Omaha area with operational detectors and a program of active data analysis that will serve as a model for statewide expansion. In the long term when there are at least 30 operating school sites, physics results from CROP will be published in refereed scientific journals and will be presented by student representatives at international cosmic-ray research conferences and by their teachers at professional meetings.

CROP has received national attention, as described in the October 1998 Physics Today article, "High School Students Will Soon Join in the Hunt for High-Energy Cosmic Rays". The main thrust of CROP is to expose its participants to the physics of cosmic rays, air showers, particle detectors, data acquisition, data analysis, the scientific method, and presentation of results. CROP also addresses physics topics which will complement the major ground-based experimental arrays (the Chicago Air Shower Array, the Pierre Auger Project, etc.) in several ways. Primary cosmic-ray energy and direction-of-origin distributions will be collected for building-sized showers $(\mathrm{E} \approx$ $10^{15} \mathrm{eV}$, high rate) using the detector array at each school and city-sized showers $\left(\mathrm{E} \approx 10^{19} \mathrm{eV}\right.$, lower rate) using time-coincidences among schools in populated areas like Omaha and Lincoln for comparison with the above experiments. In addition, CROP will make a unique scientific contribution, since CROP detector sites will eventually cover the 75,000 square mile area of the state, many times the geographic coverage of the above arrays. The sparsely spaced sites in western Nebraska will allow CROP participants to investigate very long-distance correlations, which would indicate extensive cosmic-ray bursts.

\section{The Future of CROP}

Although CROP is in its early stages, two significant milestones were passed in late 1999 and early 2000. First, CROP arranged to inherit, free of charge, a large number of retired scintillator planes, photomultiplier tubes, low voltage supplies, and high voltage supplies which were used in the now-complete Chicago Air Shower Array (CASA) experiment located in Dugway, Utah. In October 1999, four members of the CROP staff traveled to the Utah desert to recuperate sufficient detector hardware to outfit the first 10-15 schools participating in CROP. Subsequent equipment recovery trips will be made to the CASA site as needed.

Second, in early January 2000, the CROP team received word from the National Science Foundation that a major proposal submitted to the NSF Teacher Enhancement program will be funded with a starting date of June 1, 2000. The NSF grant, with a total budget of $\$ 1,342,000$ spread over 4 years, will support many of the educational aspects of CROP - in particular, 4-week summer and 2-day academic-year research experiences for teachers and students participating in CROP - as well as the technical development and optimization of the stand-alone experiment which teachers/students will install and operate at their schools and the techniques for sharing and analyzing the data over the Internet. By the end of the NSF grant period, CROP will be operating at several schools in the Lincoln/Omaha area as well as at least one school in each of the 19 ESUs across the state. These schools will serve as hubs for expansion to other schools in each region, the ultimate goal being to involve all 314 high schools in Nebraska. With the help of the University of Nebraska Foundation, private and corporate sponsors are being sought for the institutionalization of the project beyond the period of NSF funding.

\section{Bringing a National Scientific User Facility to the Student's Desktop (Myers)}

The Environmental Molecular Sciences Laboratory (EMSL) (5) at Pacific Northwest National Laboratory is a new national Department of Energy (DOE) scientific user facility supporting over 700 external users each year. It houses over 200 researchers and 70+ scientific instruments for study of the fundamental physical, chemical, and biological processes that underpin critical environmental issues. Bringing the EMSL - its environmental remediation focus, its cross-disciplinary team approach to research, its state-of-the-art instruments and computational resources, and the drive and enthusiasm of its researchers, - into the undergraduate experience can motivate students by connecting the facts in their textbooks to real-world issues and to real-world research programs.

\section{EMSL's Collaboratory Project}

The EMSL's Collaboratory project, which provides Internet collaboration technologies, including videoconferencing, collaborative instrument control, remote laboratory cameras, and electronic notebooks to a growing number of researchers, is working with academic partners to use these same technologies in a variety of education projects. A series of DOE and National Science Foundation sponsored workshops between EMSL and regional academic institutions in 1995-96 helped focus our education efforts. Participants noted that while 
collaboratories promise easier access to national facilities (e.g. the possibility for remote guest lectures and field trips), they also allow a range of participatory activities that represent a qualitative change in how national facilities and academic institutions can work together to improve science literacy and train young scientists. Bringing advanced instrumentation, data and the tools to analyze and visualize it, and the ability to talk and work with scientists to any desktop at any time for little more than the cost of existing computers and networks provides an unprecedented opportunity to link classroom studies of science with modern research practice. Workshop participants generated ideas for over twenty-five potential project with EMSL and identified three general participatory scenarios for interaction between academic institutions and research facilities, which have guided our thinking.

\section{Results and Conclusions}

Over the past five years, a number of projects in fields such as mass spectroscopy, nuclear magnetic resonance (NMR) spectroscopy, computational modeling of underground contaminant transport, environmental science, and collaboratory software development have proven the feasibility of in-depth interaction between students, educators, and EMSL researchers via the Internet. For instance, remote laboratory exercises, including a preparatory lecture and demonstration by a remote researcher, mentored remote control of an instrument to conduct experiments, and group discussion of the results and the relevance of the technique applied to important real-world issues, have allowed students to learn how multi-dimensional NMR spectroscopy is used to understand the structure of function of proteins and then perform their own experiments on small protein fragments. Similarly, faculty, researcher staff, and students have worked together via the Internet on long term research and development projects. Nominally, a student or student group conducts initial work, e.g. sample preparation and training on remote instruments, on campus during the school year, working directly with their professor and interacting via the Internet with a remote researcher. Students then travel to the laboratory for the summer for an immersive research experience, again mentored by their professor (now remote) and their summer research advisor. Finally, they return to campus, with continuing access to data, analysis software, visualization tools, and their two mentors, where they share their experience with classmates through continuing group activities. Such faculty-student-EMSL researcher teams have studied the degradation of chlorinated hydrocarbons in soils, created WWW-based tutorials on Fourier Transform Ion Cyclotron Resonance (FTICR) mass spectroscopy, and helped develop parts of EMSL's collaboratory software itself during year-long student team software projects. Faculty (and research staff) development is clearly a benefit of these $n$-way interactions that is not present in traditional student laboratory internships with faculty-staff interactions spanning students and years.

Several aspects of the EMSL Collaboratory have been critical to the success of these projects. The relatively low barriers to using the technology from anywhere at anytime make it easy to fit the schedules of researchers, faculty, and students. The existence of a multimedia electronic notebook to record, organize, and share data, notes, and visualizations, helps provide a persistent group identity and a means for group members to track progress. The public availability of the same tools for projects between academic institutions, and their availability and suitability for pure research projects allows greater returns on the investment by faculty and research staff in learning how to use them. Finally, collaboratory capabilities are provided as an EMSL-wide service, linked with active collaboratory research project. Thus, while groups can obtain support and benefit from the experience of other groups, they also have the freedom to structure projects to fit their situations and personalities, and they can expect that their feedback on the technology and requests for new functionality will be translated into improved capabilities.

The benefits to students, educators, and researchers in working "down the hall while across the country", while not quantified, are clear. Initial interactions via collaboratories are being repeated and expanded, and groups are forming long term relationships. The success of EMSL's use of collaboratory technologies fo research, as in the Virtual NMR Facility, which already supports 25\% of the users of EMSL's ten NMR spectrometers, is also driving adoption in education. Many people believe that being able to work effectively in Collaboratories will soon be an essential skill for educators and researchers and thus are beginning to consider the training in the use of Collaboratory capabilities itself a benefit of working remotely with the EMSL.

\section{Endnotes}

(1) http://sparc-1.si.umich.edu/sparc/central

(2) http://bugscope.beckman.uiuc.edu 
(3) http://chickscope.beckman.uiuc.edu

(4) http://www.physics.unl.edu/research/crop/crop.html

(5) http://www.emsl.pnl.gov:2080/docs/collab/.

\section{References}

Barstow, D. (1998). Visualizing Earth: Exploring the power of remote-sensed images and visualization technologies to help students investigate earth. In A. S. Bruckman, M. Guzdial, J. L Kolodner, \& A. Ram (Eds.), Proceedings of the International Conference of the Learning Sciences (pp. 291-293). Charlottesville, VA: AACE.

Bruce, B. C., Carragher, B. O., Damon, B. M., Dawson, M. J., Eurell, J. A., Gregory, C. D., Lauterbur, P. C., Marjanovic, M. M., Mason-Fossum, B., Morris, H. D., Potter, C. S., \& Thakkar, U. (1997). Chickscope: An Interactive MRI Classroom Curriculum Innovation for K-12, Computers and Education Journal, 29, 73-87.

Carragher, B. \& Potter, C. S. (1999). The WorldWide Laboratory: Remote and Automated Access to Imaging Instrumentation. In Impact of Advances in Computing and Communications in Chemical Sciences and Technology (National Research Council, Chemical Sciences Roundtable, pp.141-153). Washington, DC: National Academy Press.

Edelson, D. C. (1998). Matching the design of activities to the affordances of software to support inquiry-based learning. In A. S. Bruckman, M. Guzdial, J. L Kolodner, \& A. Ram (Eds.), Proceedings of the International Conference of the Learning Sciences (pp. 77-83). Charlottesville, VA: AACE.

Finholt, T. A., \& Olson, G. M. (1997). From laboratories to collaboratories: A new organizational form for scientific collaboration. Psychological Science, 8 (1), 28-36.

Horwitz, P., Schwartz, J., Kindfield, A. C. H., Yessis, L. M., Hickey, D. T., Heidenberg, A. J., \& Wolfe, E. W. (1998). Implementation and evaluation of the GenScope learning environment: Issues, solutions, and results. In A. S. Bruckman, M. Guzdial, J. L Kolodner, \& A. Ram (Eds.), Proceedings of the International Conference of the Learning Sciences (pp. 6-10). Charlottesville, VA: AACE.

Lave, J., \& Wenger, E. (1991). Situated Learning: Legitimate peripheral participation. Cambridge, England: Cambridge University Press.

Mukerji, C. (1996). The collective construction of scientific genius. In Y. Engestrom \& D. Middleton (Eds.), Cognition and Communication at Work (pp. 257-278). Cambridge, England: Cambridge University Press.

National Research Council (1993). National collaboratories: Applying information technology for scientific research. Washington, DC: National Academy Press.

Young, S.J.; Guo You Fan, G.; Hessler, D.; Lamont, S. (1996). Implementing a Collaboratory for Microscopic Digital Anatomy. International Journal of Supercomputer Applications and High Performance Computing, $10(2-3), 170-81$.

\section{Acknowledgments}

SPARC was sponsored by the National Science Foundation (IRI 9216848 and ATM 9873025).

Support of the Bugscope project and the IBM Shared University Research Program Support provided by the Illinois Consolidated Telephone Company. The NSF (9871103) and the Beckman Foundation provided the purchase of the ESEM.

The EMSL Collaboratory work was supported by the U. S. Department of Energy (DOE) through the DOE2000 program and the Distributed Collaboratory Experiment Environments (DCEE) program, both sponsored by the Mathematical, Information and Computational Sciences Division of the Office of Energy Research, and through the Laboratory Directed Research and Development program at Pacific Northwest National Laboratory (PNNL). PNNL is operated for the U. S. DOE by Battelle. The W. R. Wiley Environmental Molecular Sciences Laboratory (EMSL) is a national scientific user facility sponsored by the U.S. DOE's Office of Biological and Environmental Research and located at PNNL. 